\title{
Nanoscale architecture of graphene oxide membranes for improving dehumidification performance
}

\author{
E. A. Chernova ${ }^{1}$, D. I. Petukhov ${ }^{1}$, O. O. Kapitanova ${ }^{1}$, O. V. Boytsova ${ }^{1,2}$, A. V. Lukashin ${ }^{1}$, A. A. Eliseev ${ }^{1}$ \\ ${ }^{1}$ Lomonosov Moscow State University, Leninskiye Gory, Moscow, 119991, Russia \\ ${ }^{2}$ Kurnakov Institute of General and Inorganic Chemistry, Russian Academy of Sciences, \\ Leninsky prospect, 31, Moscow, 119991, Russia \\ wellastra@gmail.com,di.petukhov@gmail.com, olesya.kapitanova@gmail.com, boytsova@gmail.com, \\ alexey.lukashin@gmail.com, eliseev@inorg.chem.msu.ru
}

PACS 81.05.Rm, 47.56.+r

DOI 10.17586/2220-8054-2018-9-5-614-621

\begin{abstract}
Thin composite graphene oxide (GO) membranes prepared from the mixture of GO nanoflakes and nanoribbons are proposed to enhance membrane stability at elevated pressure gradients. It is shown that addition of $5-15 \%$ of GO nanoribbons to medium flake graphene oxide during deposition allows up to a $60 \%$ increase in the porosity of GO membranes. The membranes illustrate strong barrier properties to permanent gases with a permeance below $0.01 \mathrm{~m}^{3} /\left(\mathrm{m}^{2} \cdot\right.$ bar $\left.\cdot \mathrm{h}\right)$, while revealing high permeance to water vapor over $50 \mathrm{~m}^{3} /\left(\mathrm{m}^{2} \cdot \mathrm{bar} \cdot \mathrm{h}\right)$. This results in $\mathrm{H}_{2} \mathrm{O} / \mathrm{N}_{2}$ selectivity up to 12500 at water vapor fluxes over $1 \mathrm{~m}^{3} /\left(\mathrm{m}^{2} \cdot \mathrm{h}\right)$ at relative humidity of feed stream of $90 \%$. Despite $\sim 10 \%$ loss of membrane performance with addition of nanoribbons, the membranes reveal an improved stability to pressure gradients. Irreversible permeance loss of composite membranes does not exceed $10 \%$ as compared to $\sim 35 \%$ performance loss for pure medium flake graphene oxide (MFGO) after long term exposure to $0.1 \mathrm{MPa}$ pressure difference. An improved stability is invoked for the prevention of the irreversible conglomeration of GO flakes and appearance of permanent channels for water transport along the edges of nanoribbons.
\end{abstract}

Keywords: graphene oxide flakes, nanoribbons, anodic alumina, pressure stability, dehumidification, water transport.

Received: 4 August 2018

Revised: 9 September 2018

\section{Introduction}

Graphene oxide (GO) is an advanced 2D material with a variety of applications including membrane separation technologies [1]. The unique microstructure of GO is represented by a laminar assembly of stacked 2D nanosheets covered with hydrophilic oxygen-containing groups. Under standard conditions, graphene oxide shows barrier properties towards most of gases but exhibits high selectivity for water vapor, which makes it a perfect candidate for gas dehumidification processes [2-4]. Gases and vapors can migrate through graphene oxide structure by interlayer diffusion, cross-layer channels and defects in GO nanosheets [5]. The proportion between these diffusion pathways is dictated by both the GO oxidation degree and the GO microstructure friability. The last property is mainly governed by the stacking order of GO nanosheets, GO nanosheet sizes, and the method of GO membrane preparation [6]. The interrelation of GO microstructure and its separation properties under real operational conditions is the key factor for successful design of industrial GO-based membranes. It should be kept in mind, that a huge number of membrane separation processes are conducted at elevated pressures and large pressure gradients. Due to its layered structure, graphene oxide exhibits compaction under pressure gradients. This becomes a keystone problem for industrial utilization of GO membranes.

To date, a lack of attention is payed to the separation properties and stability of GO under elevated pressure. The reported results on water vapor-gas separation were obtained without absolute pressure difference between feed and permeate side [2,3]. Only few studies on permeation of liquid water across GO membranes at elevated pressure in nanofiltration processes have been published [7-10]. In [7], the decline of liquid water permeation with pressure increase was shown: at 10 bar the final water flux on a GO-based membrane was lower than $10 \%$ of its initial value. Such a strong reduction of water permeance was attributed to the compacting of the GO's laminar structure, resulting in extension of diffusion pathways for water molecules and decreasing the overall permeance. Notably, according to [7], the GO membrane can be partially regenerated after pressure exposure by drying. GO compaction is detrimental, as water molecules cannot flip through interlayer spacing and a prolonged time is required to restore the original high-water permeable GO structure.

To reduce compaction, stabilization of GO d-spacing by cross-linking can be achieved. The intercalation of cross-linking agents, cations, different nanostrands, or even single-walled carbon nanotubes has been suggested [11-14]. Alternatively, the prolonged high performance of GO membranes can be achieved by inserting 
rigid components resistive to pressure into its interlayer spacing. Such components create incompressible persistent channels for water permeance in GO nanostructure providing stable membrane performance. The best candidates for this purpose are graphene oxide nanoribbons obtained by oxidation of single walled carbon nanotubes as being most compatible with GO matrix.

Thus, here we report the results on pressure stability of composite membranes based on GO nanolfilms intercalated with GO nanoribbons deposited on anodic alumina substrates. The pressure stability of GO-based membranes is tested. It is shown that composite membranes based only on GO nanoflakes lose their water permeance with pressure, whereas addition of GO nanoribbons improves the pressure resistance of GO membranes.

\section{Experimental section}

Graphene oxide nanoflakes (MFGO) and nanoribbons (CNTGO) were prepared by oxidation of medium flake graphite and single-walled carbon nanotubes, respectively, using a modified Hummer's method [15, 16]. A detailed description of the procedure is reported in [5].

Anodic alumina membranes, with pore diameter of $\sim 100 \mathrm{~nm}$ and a thickness of $100 \mu \mathrm{m}$, were chosen as the support for GO-based composite membranes due to their excellent performance [17-19]. The AAO substrates were prepared by anodic oxidation of high purity aluminum in oxalic acid at $120 \mathrm{~V}$. To enhance the permeance of the supports, a two-stage oxidation process was employed. The detailed description of the AAO preparation is given in $[17,18,20,21]$.

To prepare composite membranes, mixed water-methanol suspensions of GO nanoflakes and GO nanoribbons in weight ratios of 20:1 (5\% CNTGO) and 7:1 (15\% CNTGO) and total GO concentration of $1 \mathrm{mg} / \mathrm{L}$ were deposited on AAO substrates using spin-coating technique at the rotation speed of $2500 \mathrm{rpm}$. The samples were denoted as MFGO-CNTGO-5 and MFGO-CNTGO-15, respectively. A reference sample of MFGO was also prepared by deposition of water-methanol suspension of pure MFGO nanoflakes.

The microstructure of AAO supports and GO-based composite membranes was studied using SEM Carl Zeiss NVision 40 (5 kV, InLens detector) electron microscopes. Raman maps of the graphene oxide selective layers were recorded by Renishaw InVia spectrometer with Leica DMLM optics $(50 \times$ objective) using $20 \mathrm{~mW} 633 \mathrm{~nm} \mathrm{He}-\mathrm{Ne}$ laser. Streamline accumulation mode with line-focused laser beam (a length of $\sim 50 \mathrm{~mm}$ and a thickness below $1 \mathrm{~mm}$ ) was used to collect the scans with total accumulation time of $200 \mathrm{~s}$ for each point. The obtained maps were treated in Wire 3.4 Renishaw software. The positions and the intensity of characteristic graphene oxide bands ( $\sim 1350$, and $\sim 1590 \mathrm{~cm}^{-1}$ ) were obtained using full profile analysis in $1000-1900 \mathrm{~cm}^{-1}$ spectral region with Pseudo-Voigt fitting of the peaks and applying 3-rd order baseline to a final tolerance factor for each spectrum of 0.01 .

The permeance of AAO supports and GO-based composite membranes was measured in two-compartment cross-flow cell using a permeance setup equipped with SLA5850 mass-flow controllers (Brooks, England), PD100-DI pressure transducers (OVEN, Russia) and T-Station 75 vacuum system (BOC Edwards, England). The measurements were performed at ambient temperature $\left(22 \pm 2{ }^{\circ} \mathrm{C}\right.$ ). Both differential (continuous pumping, flow is registered by flow controllers) and integral (closed volume, flow is registered by pressure transducers) schemes were used to measure the permeance. The water permeance was measured using a setup equipped with temperature and humidity sensors HIH-4000 (Honeywell, USA). Dew point of the sweep gas from permeate side was also registered with a dew-point hygrometer TOROS-3VY (Ukraine). The pressure in experiments was controlled using Carel SPKT00E3R pressure transducers. More details of the experiment can be found in [5].

\section{Results and discussion}

The idea for development of composite membranes comprising graphene oxide nanoflakes mixed with GO nanoribbons was originally dictated by the necessity to provide stable transport channels for water molecules, independent on pressure induced changes of GO microstructure. Indeed, rather small MFGO flakes with average sizes of $\sim 500 \mathrm{~nm}$ are extremely flexible in the layered structure, resulting in sliding and conglomeration of GO flakes under pressure gradients. Hindered intercalation of water into the interlayer space of conglomerates results in irreversible losses of membrane permeance [7]. With the introduction of GO nanoribbons with characteristic sizes of $10 \times 2000 \mathrm{~nm}$ between GO flakes we have expected the formation of voids the edges of ribbons those could not be overlayed by GO (Fig. 1). Moreover, irregular deposition of nanoribbons in between GO nanoflakes should result in increased microporosity with characteristic diameter of the pores in the order of $\sim 1 \mathrm{~nm}$. We expected that microstructural changes induced by nanoribbons will allow us to avoid the strong degradation of membranes that appear due to exclusion of water from the GO interlayer space under pressure gradients [5]. 


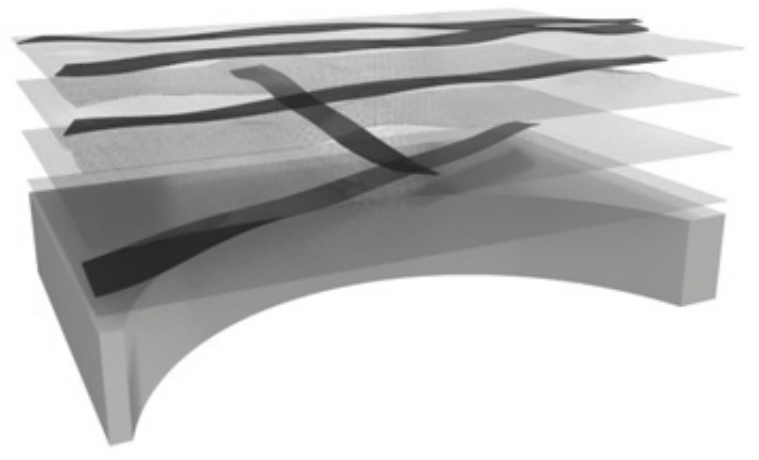

FIG. 1. Schematic illustration of the structure of MFGO-CNTGO composite membranes illustrating incompressible diffusion channels for water molecules along GO nanoribbons. Grayscale difference of nanoflakes and ribbons is given for better representation

According to SEM, addition of small amounts of graphene oxide nanoribbons $(5-15 \%)$ does not introduce critical changes to the membranes' microstructure (Fig. 2). All composite films illustrate formation of continuous GO layers on the surface of AAO substrates with characteristic thickness of $\sim 100 \mathrm{~nm}$. Detectable increase of the coating thickness from $\sim 80$ to $\sim 100 \mathrm{~nm}$ was obtained by statistical analysis of cross-sectional images. Top-view micrographs indicate remarkable difference in coatings microstructure. Additionally, a visible increase of the quantity of nanoribbons at the surface from pure MFGO to MFGO-CNTGO-15 membrane, micrographs also reveal much less pronounced corrugation of GO layers with increasing ribbons content. Such corrugations are attributed to the high flexibility of MFGO layers appearing as a result of sliding of nanoflakes during methanol evaporation. Both the increased flatness of the films and increased visible thickness can originate from decreasing density of the layers due to porosity introduced by addition of nanoribbons.

Since the porosity of films with characteristic thickness of $\sim 100 \mathrm{~nm}$ cannot currently be measured by any direct methods, we have suggested an approach based on the determination of total graphene oxide quantity from Raman scattering with subsequent normalization to statistical thickness of the layers derived by SEM. Total Raman scattering from $\mathrm{sp}^{2}$ and $\mathrm{sp}^{3}$-carbon (both $\mathrm{D}$ - and G-modes) was involved in the calculations. A film of graphene oxide produced from large-flake thermally expanded graphite with statistically measured thickness $(25 \pm 5 \mathrm{~nm})$ and porosity of $10 \%$ was used as an external standard [5]. Despite rather a large possible error produced with this semi-quantitative approach, it allowed us to determine the porosity of GO films with relative accuracy of $\pm 20 \%$ of the value.

Raman maps acquired for MFGO and MFGO-CNTGO samples illustrate diminishing of scattered signal from carbon $(\mathrm{D}+\mathrm{G}$ modes, Fig. 3(b, e), Table 1) with increasing nanoribbons content. Moreover, intensity distribution (RSD) degrades strongly in series attaining $\sim 10 \%$ for MFGO-CNTGO-15 sample. This effect is obviously connected with irregular deposition of highly anisotropic nanoribbons. The porosity of GO films extracted from an average scattering intensity normalized to film thickness illustrates strong expansion of GO film with nanoribbons content. For the MFGO-CNTGO-15 sample, the porosity attains $60 \%$ which is comparable to pure CNTGO films $(\sim 70 \%$ porosity) [5].

TABLE 1. Microstructural parameters and statistical analysis of Raman spectroscopy maps for composite membranes

\begin{tabular}{|c|c|c|c|c|c|c|}
\hline \multirow{2}{*}{ Sample } & \multirow{2}{*}{$\begin{array}{c}\text { Membrane } \\
\text { thickness } \\
\end{array}$} & \multicolumn{2}{|c|}{ D+G-mode } & \multicolumn{2}{|c|}{ D/G mode ratio } & $\begin{array}{c}\text { Derived } \\
\text { porosity, } \\
\text { (SEM }\end{array}$ \\
\cline { 4 - 7 } & $\begin{array}{c}\text { Signal } \\
\text { intensity, } \\
\text { cps· } 10^{5}\end{array}$ & RSD, \% & Average & RSD, \% & \\
\hline MFGO & $80 \pm 20$ & 3.5 & 3.5 & 2.68 & 4.4 & $10 \pm 5$ \\
\hline MFGO-CNTGO-5 & $90 \pm 20$ & 3.0 & 6.7 & 2.87 & 7.9 & $30 \pm 10$ \\
\hline MFGO-CNTGO-15 & $100 \pm 20$ & 1.8 & 8.7 & 2.94 & 13.8 & $60 \pm 10$ \\
\hline
\end{tabular}




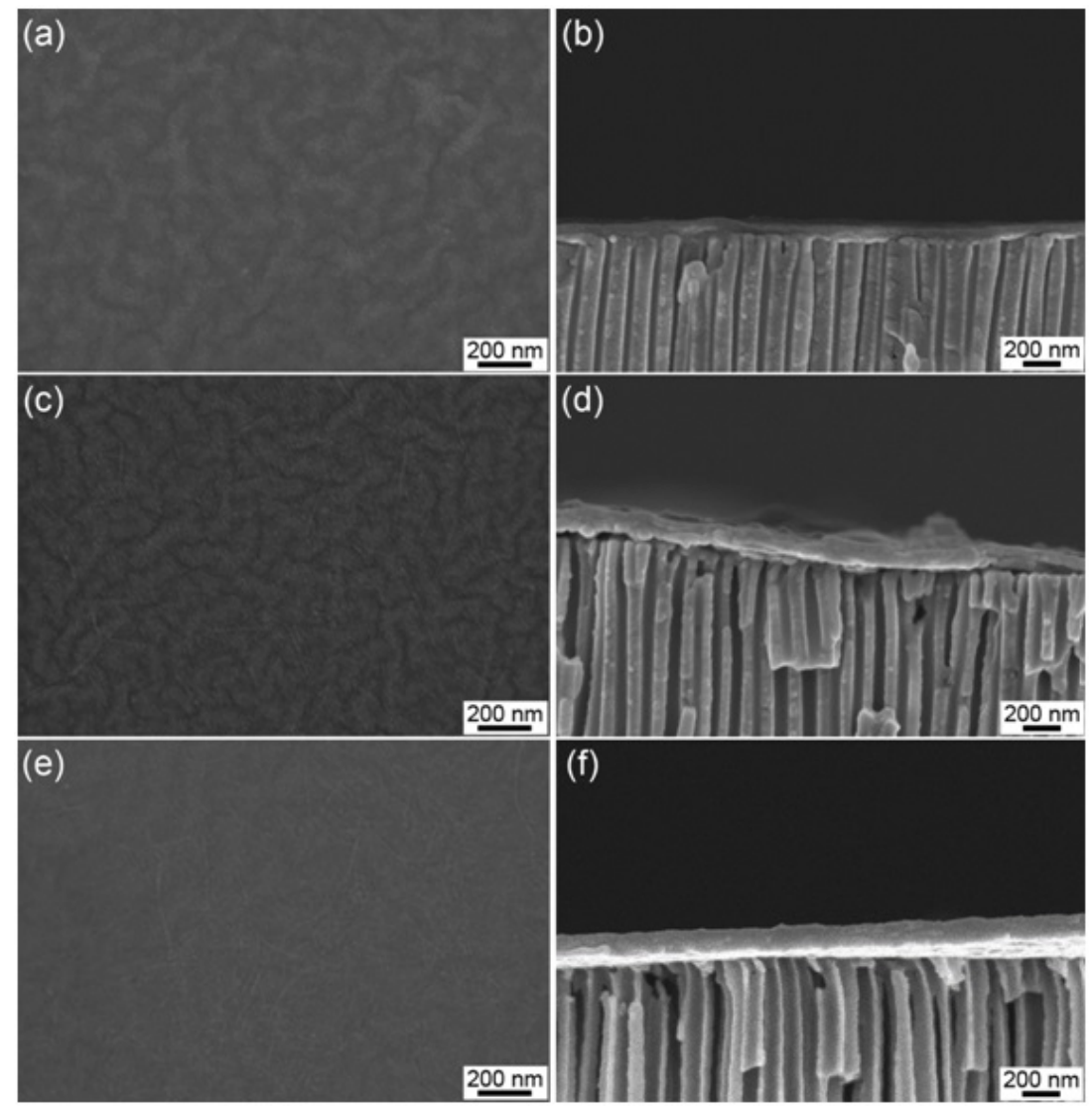

FIG. 2. Top-view and cross-sectional SEM images of composite membranes: (a, b) MFGO; (c, d) MFGO-CNTGO-5; (c, d) MFGO-CNTGO-15
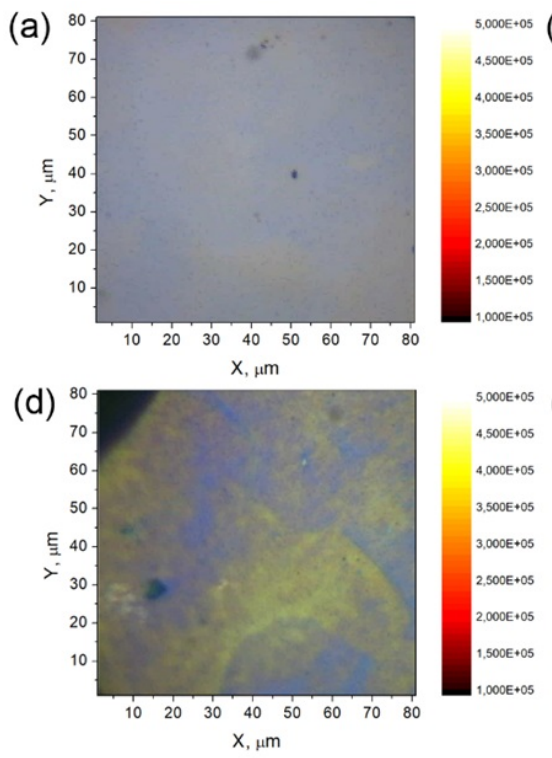
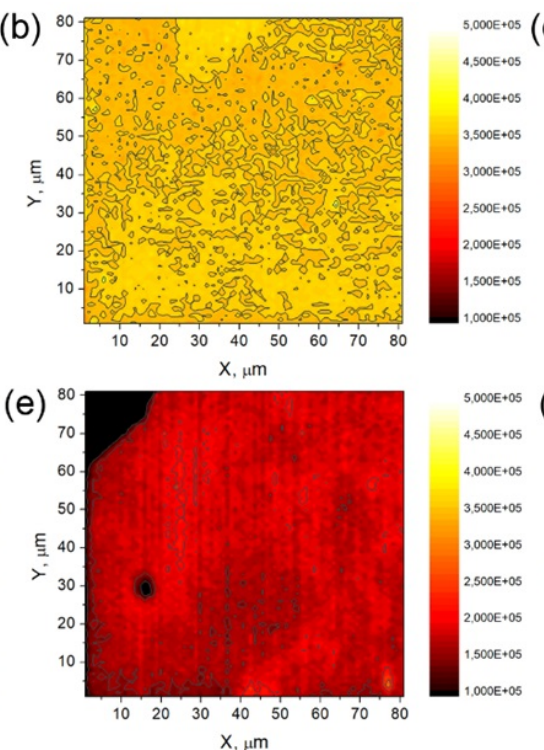
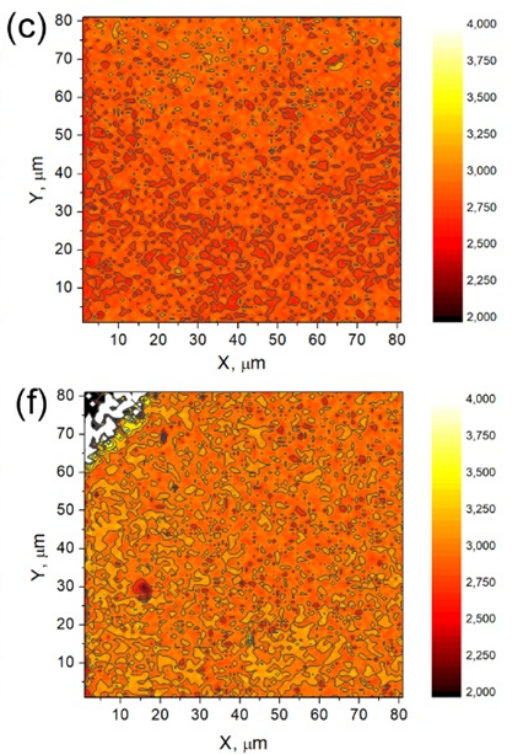

FIG. 3. Optical microscopy images (a, d) and Raman spectroscopy maps of sum of D- and G-modes intensity (b, e) and G-mode/D-mode intensity ratio (c, f) for $\operatorname{MFGO}(a, b, c)$ and MFGO-CNTGO-15 (d, e, f) samples. Black triangle in the top-left corner of (d, e, f) corresponds to an edge of sample after sample preparation for SEM 
According to Raman data, the samples illustrate minimal difference in D/G modes ratio in the films (Fig. 3(c, f); Table 1) indicating similar deposition geometry of the layers. I.e. MFGO flakes preferably lay parallel to the substrate surface forming a layered structure regardless of the presence of nanoribbons. A slight increase in both $\mathrm{D} / \mathrm{G}$ mode ratio and $\mathrm{D} / \mathrm{G}$-modes dispersion can be associated with tilting of GO flakes alongside the ribbons. Thus, introducing GO nanoribbons into MFGO films leads to an increased porosity and inhomogeneity of the films, however providing no serious effect on the layered packing of GO flakes.

Due to a rather uniform structure, the composite membranes reveal gas barrier properties towards permanent gases while maintaining high water permeance, which is favorable for dehumidification process. Typical permeabilities for membranes do not exceed 0.5 Barrer for all gases except water vapor. The membranes have a tendency to display Knudsen diffusion with the permeance of permanent gases lowering proportional to square root from molecular weight (Table 2). Such transport mechanism corresponds to gaseous diffusion through interflake edges [22]. The permeance of membranes generally increases with content of GO nanoribbons which obviously corresponds to additional pathways created by introduction of ribbons to the layered structure.

TABLE 2. Gas permeance of composite membranes

\begin{tabular}{|c|c|c|c|c|c|c|c|}
\hline \multirow{2}{*}{ Sample } & \multicolumn{6}{|c|}{ Permeance, $1 /\left(\mathrm{m}^{2} \cdot\right.$ bar.h } & \multirow{2}{*}{$\begin{array}{c}\mathrm{H}_{2} \mathrm{O} / \mathrm{N}_{2} \text { selectivity } \\
\text { at } \mathrm{RH} 90 \%\end{array}$} \\
\cline { 2 - 7 } & $\mathrm{CH}_{4}$ & $\mathrm{~N}_{2}$ & $\mathrm{O}_{2}$ & $\mathrm{CO}_{2}$ & $\mathrm{C}_{4} \mathrm{H}_{10}$ & $\mathrm{H}_{2} \mathrm{O}$ at $\mathrm{RH} 90 \%$ & 14650 \\
\hline MFGO & 5.2 & 3.9 & 3.7 & 3.2 & 2.1 & 63050 & 12520 \\
\hline MFGO-CNTGO-5 & 6.1 & 4.4 & 3.96 & 3.7 & 2.5 & 55100 & 3220 \\
\hline MFGO-CNTGO-15 & 16 & 9.7 & 8.9 & 8.3 & 4 & 51500 & \\
\hline $1 / \sqrt{M}$, mole $^{0.5} / \mathrm{kg}^{0.5}$ & 7.90 & 5.98 & 5.59 & 4.77 & 4.15 & 7.45 & \\
\hline
\end{tabular}

Notably, the permeance of composite membranes for permanent gases was found to be two orders of magnitude lower as compared to pure CNTGO membranes, revealing much lower quantity of direct pathways for gaseous transport. Moreover, $\mathrm{N}_{2}$ permeance also diminishes as compared to thin $(30 \mathrm{~nm})$ MFGO film, which is accounted for by an overall increase of the diffusion lengths and blocking of the diffusion pathways through interflake edges due to increased film thicknesses.

Diminishing the permeance for permanent gases is only partly accompanied by permeance losses for water vapor. The MFGO membrane, with a thickness of $80 \mathrm{~nm}$, exhibits water vapor permeance over $60 \mathrm{~m}^{3} /\left(\mathrm{m}^{2} \cdot \mathrm{bar} \cdot \mathrm{h}\right)$, compared to $\sim 100 \mathrm{~m}^{3} /\left(\mathrm{m}^{2} \cdot\right.$ bar.h) for $30 \mathrm{~nm}$ membrane. Nonlinear behavior of permeance can be attributed to the changes in an equilibrium interlayer distance of GO in the surface layers at different partial water pressures on permeate sides of membrane [5]. With increased nanoribbon content, the water vapor permeance slightly decreases in all the humidity range (Fig. 4). In all likelihood, a portion of rather large voids emerges in GO membrane volume due to crossing of nanoribbons. Such pores can persist as gas-filled, even at high vapor pressures, which prevents capillary transport of liquefied water through membrane. In concert with enhanced transport of permanent gases, this results in loss of selectivity of membranes. However absolute selectivities still exceed 3000 at water permeance of $\sim 1.0 \mathrm{~m}^{3} /\left(\mathrm{m}^{2} c d o t h\right)$, remaining sufficient for technological dehumidification process.

Notably, the humidity dependence of water permeance of MFGO is reproduced well by composite membranes (Fig. 4). This indicates that general permeation mechanism for water molecules in GO films is irrespective of the porosity introduced by GO nanorribbons. This fact supports the major role of cross-layer transport of water molecules through the defects in the graphene layer $[5,23]$.

The pressure stability of membranes was tested under stepwise elevation of feed pressure followed by exposure to pressure gradient and stepwise pressure release. Total experiment duration for each membrane exceeded $20 \mathrm{~h}$. The reference MFGO membrane illustrates low water permeance stability under transmembrane pressure. Initially, being the most permeable to water vapor, this membrane loses $25 \%$ of permeance at first $0.02 \mathrm{MPa}$ pressure step. At $0.1 \mathrm{MPa}$ water permeance falls to $\sim 25 \mathrm{~m}^{3} /\left(\mathrm{m}^{2} \cdot \mathrm{bar} \cdot \mathrm{h}\right)$. The reduction of GO permeance stays in agreement with earlier studies explaining the effect by the pressure-driven ousting of water molecules from the interlayer space of GO induced by bearing reaction of the support [5]. However, at pressure release, MFGO membrane exhibits a strong hysteresis, regenerating to $\sim 40 \mathrm{~m}^{3} /\left(\mathrm{m}^{2} \cdot\right.$ bar.h $)$ only, thus loosing $\sim 35 \%$ of the initial permeance after pressure exposure. This is obviously caused by irreversible changes in membrane microstructure, attributed to sliding and conglomeration of flexible GO flakes under transmembrane pressure. Those changes hinder further intercalation of water into the interlayer space of GO conglomerates resulting in membrane permeance losses. 


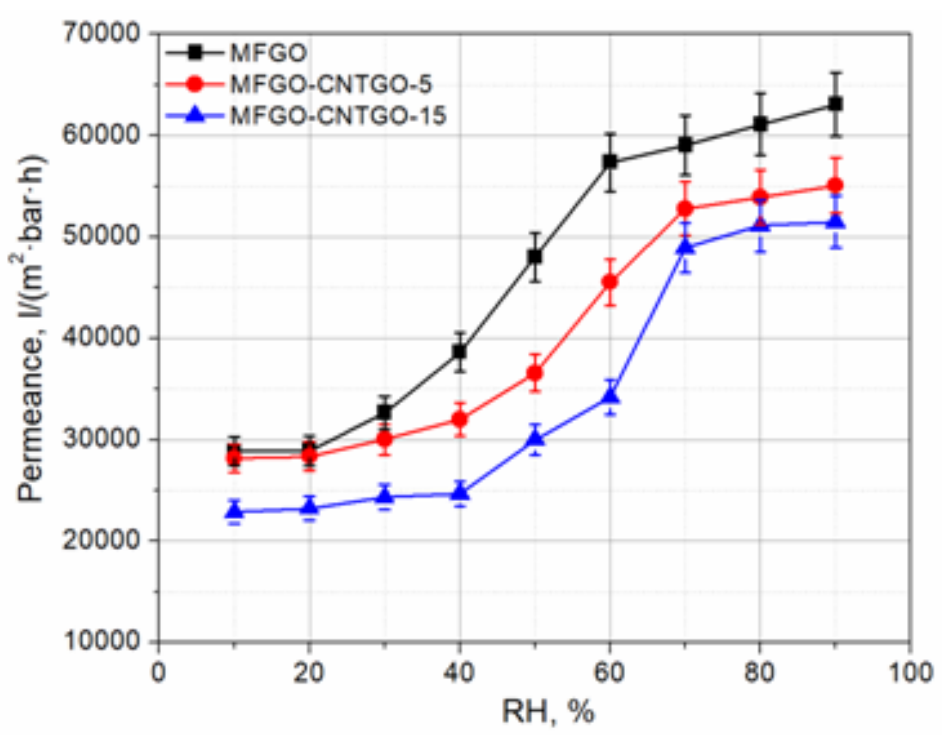

FIG. 4. The dependencies of water vapor permeance on feed gas humidity for composite membranes. Both sides of membrane were kept at atmospheric pressure, while permeate side was swept with dry He stream

Composite membranes exhibit similar behavior under an elevated pressure. At high pressure difference the permeance of these membranes becomes nearly equal to that of pure MFGO membrane, reflecting the pressuredriven ousting of water and permeance limitation by surface layers. A small permeance gain is achieved for the MFGO-CNTGO-5 sample at a transmembrane pressure of $0.1 \mathrm{MPa}$. On pressure release, both membranes restore permeance close to initial values with irreversible losses not exceeding $10 \%$. The losses are reduced with increasing the content of nanoribbons, indicating improved stability of the structure. Notably, both the composite membranes illustrate higher water permeance after pressure exposure as compared to pure MFGO membrane. These facts prove applicability of the proposed strategy for stabilization of GO structure and allow quick restoration of GO permeability through the introduced transport channels after membrane compression.

The proposed approach does not require any expensive linking agents or any special membrane modification steps and can be readily utilized at an industrial scale for the production of GO dehumidification membranes. We also believe the methodology is further applicable for the creation of permanent flexible transport channels in layered membranes for other challenging issues of membrane science and technology.

\section{Conclusions}

Thus the proposed strategy for the creation of composite membranes comprising graphene oxide nanoflakes mixed with GO nanoribbons allows improvement of membrane resistance towards elevated pressure gradients, while maintaining high performance and selectivity of graphene oxide. The insertion of $5-15 \mathrm{wt} \%$ of GO nanoribbons into medium flake graphene oxide during deposition gave rise to an increase in the porosity of GO membranes up to $60 \%$. The obtained composite membranes possess high permeance towards water vapor (over $50 \mathrm{~m}^{3} /\left(\mathrm{m}^{2} \cdot \mathrm{bar} \cdot \mathrm{h}\right)$ ), while exhibiting strong barrier properties to permanent gases, resulting in $\mathrm{H}_{2} \mathrm{O} / \mathrm{N}_{2}$ selectivity up to 12,500 at water fluxes over $1 \mathrm{~m}^{3} /\left(\mathrm{m}^{2} \cdot \mathrm{h}\right)$ at the relative humidity of feed stream of $90 \%$. The GO nanoflake-nanoribbons network reveals enhanced stability to pressure gradients but at a cost of $\sim 10 \%$ loss of membrane performance. Irreversible permeance loss of composite membranes based on the MFGO-CNT mixture does not exceed $10 \%$ as compared to $\sim 35 \%$ performance loss for pure MFGO after long term exposure to $0.1 \mathrm{MPa}$ pressure difference. Enhanced pressure stability is attributed to the prevention of the irreversible conglomeration of GO flakes and the formation of permanent flexible channels for water transport across the edges of nanoribbons. This simple approach permits the wide application of graphene oxide in separation technologies. 

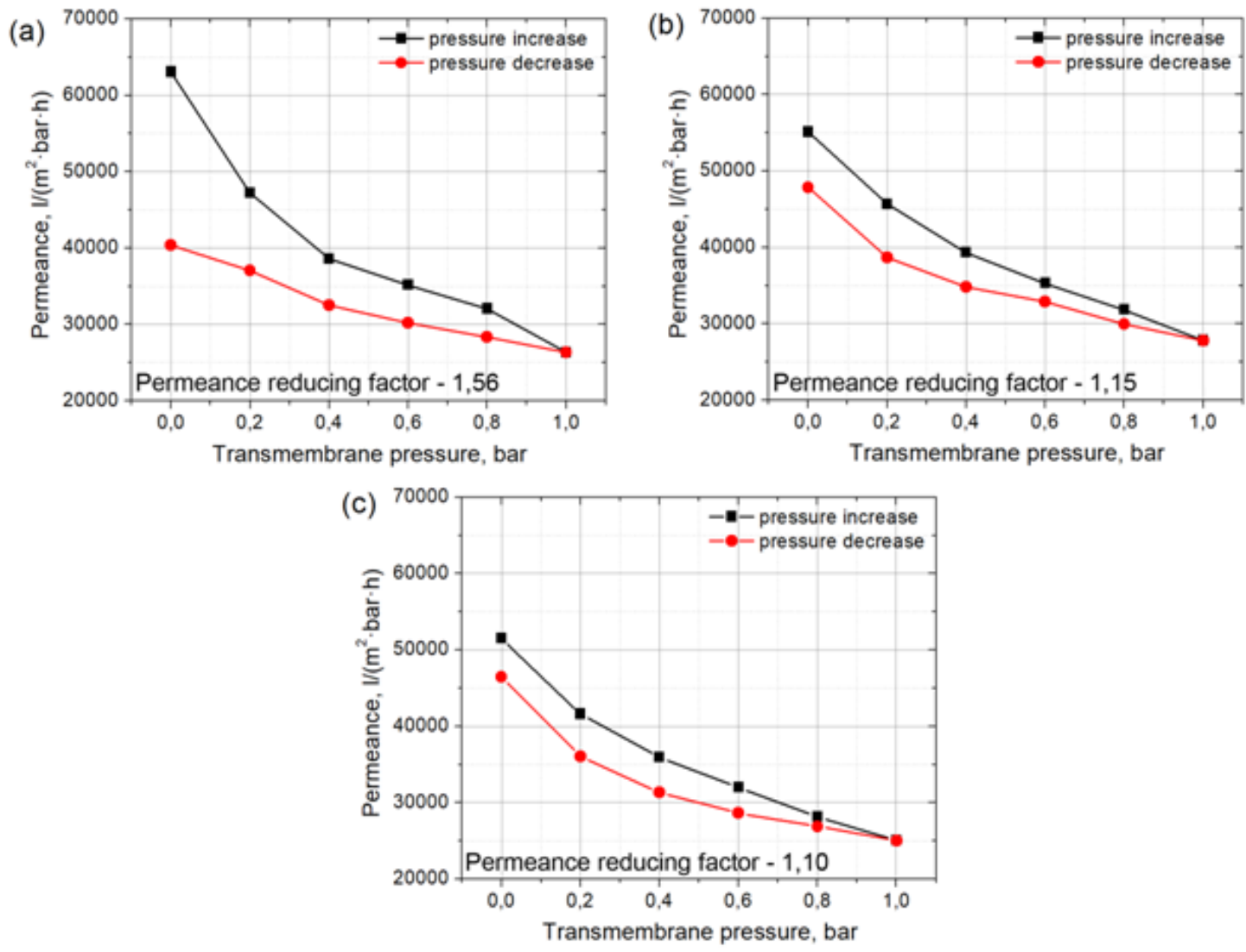

FIG. 5. Pressure dependencies of water vapor permeance for graphene oxide membranes (a) MFGO; (b) MFGO-CNTGO-5; (c) MFGO-CNTGO-15. The black curve corresponds to increasing pressure gradient from standard conditions, red curve - to pressure gradient decrease after exposure to $0.1 \mathrm{MPa}$ pressure difference. A feed stream with $90 \% \mathrm{RH}$ at absolute pressures of 1 - 2 bar was used in experiments. Absolute permeate pressure was always maintained at atmospheric pressure with He sweep gas

\section{Acknowledgements}

The work is supported by the Ministry of education and science of the Russian Federation within a Federal Targeted Programme for "Research and Development in Priority Areas of Development of the Russian Scientific and Technological Complex for 2014-2020" (Agreement No. 14.604.21.0177, unique Project Identification RFMEFI60417X0177).

\section{References}

[1] Ma J., Ping D., Dong X. Recent Developments of Graphene Oxide-Based Membranes: A Review. Membranes,2017, 7 (3), P. $52-81$.

[2] Shin Y., Liu W., et al. Graphene oxide membranes with high permeability and selectivity for dehumidification of air. Carbon, 2016, 106, P. 164-170.

[3] Athanasekou C., Pedrosa M., et al. Comparison of self-standing and supported graphene oxide membranes prepared by simple filtration: Gas and vapor separation, pore structure and stability. Journal of Membrane Science, 2017 522, P. 303-315.

[4] Nair R.R., Wu H.A., et al. Unimpeded Permeation of Water Through Helium-Leak-Tight Graphene-Based Membranes. Science, 2012, 335 (6067), P. 442-444.

[5] Petukhov D.I., Chernova E.A., et al. Thin graphene oxide membranes for gas dehumidification. Journal of Membrane Science, (submitted), 2018.

[6] Nandy K., Palmeri. M., et al. Stop Motion Animation Reveals Formation Mechanism of Hierarchical Structure in Graphene Oxide Papers. Advanced Materials Interfaces, 2016, 3 (6), 1500666.

[7] Chong J.Y., Wang B., Mattevi C., Li K. Dynamic microstructure of graphene oxide membranes and the permeation flux. Journal of Membrane Science, 2018, 549, P. 385-392.

[8] Yi H., Zhen X., Chao G. Ultrathin Graphene Nanofiltration Membrane for Water Purification. Advanced Functional Materials, 2013, 23 (29), P. 3693-3700. 
[9] Wei Y., Zhang Y., et al. Declining flux and narrowing nanochannels under wrinkles of compacted graphene oxide nanofiltration membranes. Carbon, 2016, 108, P. 568-575.

[10] Wei N., Peng X., Xu Z. Understanding Water Permeation in Graphene Oxide Membranes. ACS Applied Materials \& Interfaces, 2014, 6 (8), P. 5877-5883.

[11] Huang K., Liu G., Jin W. Vapor transport in graphene oxide laminates and their application in pervaporation. Current Opinion in Chemical Engineering, 2017, 16, P. 56-64.

[12] Han Y., Jiang Y., Gao C. High-Flux Graphene Oxide Nanofiltration Membrane Intercalated by Carbon Nanotubes. ACS Applied Materials \& Interfaces, 2015, 7 (15), P. 8147-8155.

[13] Hung W.-S., Tsou C.-H., et al. Cross-Linking with diamine monomers to prepare composite graphene oxide-framework membranes with varying d-spacing. Chemistry of Materials, 2014, 26 (9). P. 2983-2990.

[14] Huang H., Song Z., et al. Ultrafast viscous water flow through nanostrand-channelled graphene oxide membranes. Nature Communications, 2013, 4, P. 2979.

[15] Marcano D.C., Kosynkin D.V, et al. Improved Synthesis of Graphene Oxide. ACS Nano, 2010, 4 (8), P. $4806-4814$.

[16] Kosynkin D.V., Higginbotham A.L, et al. Longitudinal unzipping of carbon nanotubes to form graphene nanoribbons. Nature, 2009, 458, P. 872-876.

[17] Petukhov D.I., Napolskii K.S., et al. Comparative study of structure and permeability of porous oxide films on aluminum obtained by single- and two-step anodization. ACS Applied Materials and Interfaces, 2013, 5 (16), P. 7819-7824.

[18] Petukhov D.I., Napolskii K.S., Eliseev A.A. Permeability of anodic alumina membranes with branched channels. Nanotechnology, 2012, 23 (33), P. 335601.

[19] Petukhov D.I., Eliseev A.A. Gas permeation through nanoporous membranes in the transitional flow region. Nanotechnology, 2016, 27 (8), P. 85707.

[20] Napolskii K.S., Roslyakov I.V, et al. Origin of long-range orientational pore ordering in anodic films on aluminium. Journal of Materials Chemistry, 2012, 22 (24), P. 11922-11926.

[21] Petukhov D.I., Buldakov D.A., et al. Liquid permeation and chemical stability of anodic alumina membranes. Beilstein Journal of Nanotechnology, 2017, 8, P. 561-570.

[22] Ibrahim A., Lin Y.S. Gas permeation and separation properties of large-sheet stacked graphene oxide membranes. Journal of Membrane Science, 2018, 550, P. 238-245.

[23] Eliseev A.A., Kumskov A.S., et al. Mass Transport through Defects in Graphene Layers. The Journal of Physical Chemistry C, 2017, 121 (42), P. 23669-23675. 